\title{
A Review Article on Etiopathogenesis of Osmf
}

\author{
Dr. Veenita Singh,Dr. Mohit Srivastava,Dr. Rajesh Roshan,Dr. Harsimrat \\ Dhingra,Dr. Tushar Goel,Dr. Rahul Mishra
}

\begin{abstract}
Areca nut chewing is a great hazard and related to oral mucosa lesion in large population worldwide. In India pan masala, gutkha and mawa chewing is quite prevalent. In some sections of society, quid chewing is even part of the social culture. Areca nut which is most commonly associated with development of OSMF contains various alkaloids which interfere with molecular process of deposition and/or degradation of extracellular matrix molecule such as collagen causing imbalance in normal process. The various pathological effects of these alkaloids are:

1. Reduced phagocytosis of collagen by fibroblast.

2. Up and down regulation of copper dependent enzyme lysyl oxidase, matrix metallo proteinase and tissue inhibition of matrix metallo proteinase .

3. Increased levels of cytokines in lamina propria.

Various studies done in past put OSMF as premalignant disorder (PMDs). Understanding the etiopathology of OSMF may help to revert the risk factors so as to minimize the chance of development of disease which is incorporated in primordial prevention of disease.
\end{abstract}

\section{Introduction}

OSMF is an insidious, chronic disease affecting any part of the cavity and sometimes the pharynx. Occasionally it is preceded by and/or associated with vesicle formation and is always associated with a juxtaepithelial inflammatory reaction followed by fibroelastic change of lamina propria, with epithelial atrophy leading to stiffness of the oral mucosa and causing trismus and inability to eat. Oral submucous fibrosis (OSMF) is a premalignant condition mainly associated with the practice of chewing betel quid containing areca nut, a habit common among South Asian people. It is characterized by inflammation, increased deposition of submucosal collagen and formation of fibrotic bands in the oral and paraoral tissues, which increasingly limit mouth opening ${ }^{3}$.

Various hypotheses were put forward which suggest a multifactorial origin for the etiology of OSMF. The etiological factors include local irritants such as chili consumption, areca nut chewing, tobacco smoking and chewing. Systemic factors include anemia (iron deficiency), vitamin deficiencies (B-complex and folate) together with the malnourished state (protein deficiency), genetic predisposition to the disease and autoimmunity. The global incidence in 1996 of submucous fibrosis was estimated as 2.5 million individuals ${ }^{5}$. In Indian populations the prevalence is $5 \%$ for women and $2 \%$ for men. Age groups below 20 years are more often contracted with submucous fibrosis ${ }^{6-11}$. The aim of the present review is to understand the etiology and pathogenesis of OSMF with special emphasis on areca nut \& its constituents.

\section{Etiopathogenesis}

Arecanut

Areca nut constitutes of alkaloids, flavonoids and other trace elements like copper. The major alkaloids found in areca nut are arecoline, arecaidine, guvacine, guvacoline which cause fibroblastic proliferation and increased collagen formation. Hydrolysis of arecoline produces arecaidine that has pronounced effects on fibroblasts12. The stimulation of fibroblasts is greater with arecaidine, and there was a concentration-dependent stimulation of collagen synthesis when fibroblasts were exposed to both arecloine and arecaidine, addition of slaked lime calcium hydroxide $(\mathrm{Ca}(\mathrm{OH}) 2)$ to areca nut in pan facilitates hydrolysis of arecoline to arecaidine making this agent available in the oral environment and hence causing greater stimulation of fibroblast 13.

Flavonoid component of areca nut have some direct influence on collagen metabolism, enhancing the cross linking of collagen fibers and hence raise the lysyl-oxidase (LOX) activity14,15. The LOX activity is important for formation of insoluble collagen due to cross-linking. The process of cross-linking gives tensile strength and mechanical properties to the fibers as well as makes the collagen fibers resistant to proteolysis. The LOX is an essential enzyme for final processing of collagen fibers into a stabilized covalently cross-linked mature fibrillar form that is resistant to proteolysis 16.

Copper causes up-regulation of lysyl-oxidase (LOX) enzyme. The LOX is dependent on copper for its functional activity17. During the biosynthesis of LOX, copper is incorporated into LOX. LOX also contains a co-factor lysine tyrosylquinone (LTQ), a covalently bound carbonyl prosthetic group18. The LTQ is essential in 
the formation of cross-links in the collagen fibers for the reaction mechanism of LOX19. Copper has been suggested to play a structural role in stabilizing the LTQ19. During the process of cross-linking, copper plays an important role in reoxidizing the reduced enzyme facilitating the completion of the catalytic cycle. Areca nuts have been shown to have a high copper content, and chewing areca nuts for 5-30 min significantly increases soluble copper levels in oral fluids. This increased level of soluble copper could act as an important factor in OSF by stimulating fibrogenesis through up-regulation of LOX activity20,21.

To summarize the action of the major etiological factor arecanut in the pathogenesis of OSF, the alkaloids cause the stimulation of fibroblast and gene expression hence leading to increased collagen production. The flavanoids increase the cross-linking of collagen and copper causes the up-regulation of lysyl oxidase and hence stimulating fibrogenesis.

\section{Molecular pathogenesis:}

Of all of the growth factors, none has been found to have the diversity of effects on extracellular matrix (ECM) ascribed to transforming growth factor- $\beta$ (TGF- $\beta$ ). This peptide plays a critical role not only in synthesis and degradation of ECM but also in response of cells to ECM mediated through integrin receptors; moreover, specific components of the ECM, in turn, can both deliver TGF- $\beta$ and regulate its activity. ${ }^{23,24}$ Over a period of time, due to persistent habit, chronic inflammation sets in at the site. Initial irritation leads to further atrophy and ulceration of the mucosa. It can thus be considered that induction of oral mucosal inflammation by betel quid ingredients is a critical event in the pathogenesis of OSMF. Cytokines like interlukin-6 (IL-6), tumor necrosis factor (TNF), interferon- $\alpha(\mathrm{INF}-\alpha)$ etc. and growth factors like TGF- $\beta$ are synthesized at the site of inflammation. TGF- $\beta 1$ is

a key regulator of extra cellular matrix (ECM) assembly and remodeling. The action of TGF- $\beta$ on the genes implicated in the formation and degradation of the ECM is mostly exerted at the transcriptional level through ill defined intracellular pathways. TGF- $\beta$ increases the collagen production and decreases the collagen degradation.

\section{Role of Genetic and immunological factors:}

Gene-gene interaction analysis revealed that XRCC3 Thr 241 Met had the largest univariate effect followed by XRCC3 Thr 241 Met -NAT2 A857G in men that presents a highly synergistic interaction as one of the potential combinations of single nucleotide polymorphisms (SNPs) to increase the risk of OSMF in men if exposed to arecanut or smokeless tobacco usage. ${ }^{25}$ Connective tissue growth factor (CTGF/CCN2) is associated with many human fibrotic disorders and was found to overexpress in OSMF. Microtrauma could lead to the release of thrombin. Thrombin produced by microtrauma may contribute to the pathogenesisof OSMF by upregulating CCN2 expression. This effect could be mediated by protease-activated receptor-1, reactive oxygen species, apoptosis signal-regulating kinase 1, and c-Jun $\mathrm{NH}(2)$-terminal kinase pathways and prevented by epigallocatechin-3-gallate. $^{26}$

Collegen-related genes COL1A2, COL3A1, COL6A1, COL6A3 and COL7A1 have been identified as targets of transforming growth factor-b (TGF- b) and induced fibroblasts at an early stage of the disease. 30 These genes play an important role in the homeostasis of collagen in the body. The genetic modulation of different enzymes such as collagenases and lysyl oxidase together with cytokines, namely TGF-b has been implicated in this context. The transcriptional activation of procollagen genes by TGF-b may contribute to increased collagen levels and hence increased expression of procollagen genes and thereby contributing to increased collagen level in OSF.23 Chiu et al. analyzed two groups of betel chewers, one with OSF and the other without in order to compare the association of OSF and polymorphisms of six collagen related genes.30 They used PCR-based restriction fragment length polymorphism assays to determine the genotypes of the six collagen-related genes situated on different chromosomes and found that the genotypes associated with the highest OSF risk for collagen 1A1, collagen 1A2, collagenase-1, transforming growth factor beta1, lysyl oxidase, and cystatin $\mathrm{C}$, an increased risk of OSF was noted with an increasing number of high-risk alleles for those with both high and low exposures for betel quid. The cell selection mechanism of oral fibroblasts is proposed to explain the effect of the modification of cumulative betel quid exposure on the risk profiles of collagen-related genes, susceptibility to OSF could involve multigenic mechanisms modified by the betel quidexposure dose.

\section{Role of Heat shock proteins (HSP)}

HSP4, is a $47 \mathrm{kDa}$ collagen-binding heat shock protein (HSP), known as a molecular chaperone belongs to the serine protease inhibitor (serpin) superfamily and is involved in the synthesis, processing, and assembly of various collagens. Shung et al., first found that arecoline has the tendency to upregulate HSP47 mRNA expression in human buccal mucosal fibroblasts (BMFs). HSP47 and hence cause the accumulation of collagen in oral mucosal connective tissue and cause OSF.37 


\section{Conclusion}

OSMF is multifactorial involving multigenic mechanisms modified by the betel quid exposure dose. Epidemiological evidences suggest well established relationship between areca nut and OSMF. The chemical constituents of areca nut have been identified as arecoline, arecaidine, guvacine, guvacoline, catachins and tannin in biochemical studies. These chemical constituents of areca nut stimulate fibroblast proliferation leading to collagen synthesis. Also molecular pathogenesis suggests the role of growth factors in inducing fibrotic tissue formation. In conclusion, further studies are required to establish the role of betel nut in addition to genetic factors and autoimmunity in pathogenesis of OSMF.

\section{References}

[1]. Pindborg J, Sirsat S. Oral Submucous Fibrosis. Oral Surgery, Oral Medicine, and Oral Pathology 1966;22(6):764

[2]. Rajendran R. OSMF: etiology, pathogenesis and future research. Bulletin of the World Health Organization 1994;72(6):985-96

[3]. Auluck A, Rosin MP, Zhang L, Sumanth KN.Oral submucous fibrosis, a clinically benign but potentially malignant disease: report of 3 cases and review of the literature. JCan Dent Assoc2008 Oct; 74(8):735-40

[4]. Auluck A, Rosin MP, Zhang L, Sumanth KN.Oral submucous fibrosis, a clinically benign but potentially malignant disease: report of 3 cases and review of the literature. JCan Dent Assoc 2008 Oct; 74(8):735-40

[5]. Cox SC, Walker DM. Oral submucous fibrosis: A review. Aust Dent J 1996; 41: 294-299.

[6]. Su I.P. Idiopathic scleroderma of the mouth. Report of three cases. Archives of otolaryngology 1954; 59: 333-2.

[7]. Behl PN. Practice of dermatology. Allied publishers private limited, Bombay. 1962.

[8]. Rao ABN. Idiopathic palatal fibrosis. British journal of surgery 1962; 50: 23-25.

[9]. Cox SC, Walker DM. Oral submucous fibrosis: A review. Aust Dent J 1996; 41: 294-299.

[10]. Thavarajah R, Rao A, Raman U et al. Oral lesions of 500 habitual psychoactive substance users in Chennai. India. Arac Oral Biol 2006; 51: 512-519.

[11]. Jontell M, Holmstrup P. Red and white lesions of oral mucosa. In: Greenberg SM, Brightman JV Burkets oral medicine.11th edition. Hamilton: BC Decker Inc; 2008. p 88-89.

[12]. Harvey W, Scutt A, Meghji S, Canniff P. Stimulation of human buccal mucosa fibroblasts in vitro by betel nut alkaloids. Arch Oral Biol 1986; 31: 45-49.

[13]. Nieschultz V, Schmersahl P. Zur Phamakologie der Wirkstoffe des Betels Umwenlung des arecolin in arecadin. ArzneimittelForsch 1968; 18: 222-225.

[14]. DiSilvestro RA, Harris ED. Evaluation of catechin action on lysyl oxidase activity in aortic tissue. Biochem Pharmacol 1983; 32 : 343-346.

[15]. Cetta G, Gerzeli G, Quartieri A, et al. Protective effect of flavonoids on the collagen of lathyritic rats. Experientia 1971; 27: 10468.

[16]. Rajalalitha P, Vali S. Molecular pathogenesis of oral submucous fibrosis-a collagen metabolic disorder. J Oral Pathol Med 2005; 34: 321-328.

[17]. Rucker RB, Romero-Chapman N, Wong T, et al. Modulation of lysyl oxidase by dietary copper in rats. J Nutr 1996; 126: 51-60.

[18]. Smith-Mungo LI, Kagan HM. Lysyl oxidase: properties, regulation and multiple functions in biology. Matrix Biol 1998; 16: 387398.

[19]. Williamson PR, Kagan HM. Reaction pathway of bovine aortic lysyl oxidase. J Biol Chem 1986; $261: 9477-82$.

[20]. Tang C, Klinman JP. The catalytic function of bovine lysyl oxidase in the absence of copper. J Biol Chem 2001; 276: 30575-30578.

[21]. Trivedy C, Meghji S, Warnakulasuriya KA, Johnson NW, Harris M. Copper stimulates human oral fibroblasts in vitro: A role in the pathogenesis of oral submucous fibrosis. J Oral Pathol of Med 2001; 30: 465-70.

[22]. Trivedy CR, Warnakulasuriya KA, Peters TJ, Senkus R, Hazarey VK, Johnson NW. Raised tissue copper levels in oral submucous fibrosis. J Oral Pathol Med 2000; 29: 22-48.

[23]. Silverio-Ruiz KG, Martinez AE, Garlet GP, Barbosa CF, Silva JS, et al. Opposite effects bFGF and TGF-beta on collagen metabolism by human periodontal ligamentfibroblasts.Cytokine 2007;39: 130-7.

[24]. Shah N, Sharma PP. Role of Chewing and Smoking habits in the etiology of Oral submucous fibrosis: a case-control study; J Oral Pathol Med 1998; 27: 475-9.

[25]. Mukherjee S, Bhowmik AD, Roychoudhury P, Mukhopadhyay K, Ray JG, IChaudhuri K, et al.Association of XRCC1, XRCC3, and NAT2 polymorphisms with the risk of oral submucous fibrosisamong eastern Indian population.J Oral Pathol Med 2012;41(4):292-302.

[26]. Chang JZ, Yang WH, Deng YT, Chen HM, Kuo MY.Thrombin-stimulated connective tissue growth factor (CTGF/CCN2) production in human buccal mucosal fibroblasts: Inhibition by epigallocatechin-3-gallate.Head Neck2011 Sep 22. doi: 10.1002/hed.21863

[27]. Dyavanagoudar S N. Oral Submucous Fibrosis: Review of Etiopathogenesis. J Cancer Science \& Theraphy 2009; 1: $72-77$. 